\title{
A WEIGHTED HYPERPLANE MEAN ASSOCIATED WITH HARMONIC MAJORIZATION IN HALF-SPACES
}

\author{
by D. H. ARMITAGE
}

(Received 12th December 1984)

\section{Introduction and main results}

The purpose of this paper is to introduce a new kind of weighted hyperplane mean for subharmonic functions and to use this mean in giving results on the harmonic majorization of subharmonic functions of restricted growth in half-spaces.

An arbitrary point of the Euclidean space $\mathbf{R}^{n+1}$, where $n \geqq 1$, will be denoted by $M=(X, y)$ where $X=\left(x_{1}, \ldots, x_{n}\right) \in \mathbf{R}^{n}$ and $y \in \mathbf{R}$. We write

$$
|X|=\left(x_{1}^{2}+\cdots+x_{n}^{2}\right)^{\frac{1}{2}}, \quad|M|=\left(|X|^{2}+y^{2}\right)^{\frac{1}{2}}
$$

and, in the sense of Lebesgue, $d X=d x_{1} \ldots d x_{n}$. Throughout this paper $a$ and $b$ will be real numbers such that $0 \leqq a<b$ and

$$
D_{a}=\left\{M \in \mathbf{R}^{n+1}: y>a\right\}, \quad \Omega_{a, b}=\left\{M \in \mathbf{R}^{n+1}: a<y<b\right\}
$$

If $f$ is a non-negative Lebesgue measurable function on $\mathbf{R}^{n} \times\{y\}$, where $y>a$, let

$$
\Psi_{a}(f, y)=(y-a)^{-n-1} \int_{\mathbf{R}^{n}}\{1+|X| /(y-a)\}^{\frac{1}{2}(1-n)} e^{-\pi|X| /(y-a)} f(X, y) d X .
$$

If $f$ takes values of both signs, we write

$$
\Psi_{a}(f, y)=\Psi_{a}\left(f^{+}, y\right)-\Psi_{a}\left(f^{-}, y\right),
$$

provided at least one of the terms on the right-hand side is finite.

The weighted mean $\Psi_{a}$ is related to the mean introduced by Brawn in his study of subharmonic functions in strips [4], and this paper depends upon his work. Our theorems, however, are more closely analogous to those of Kuran [9] on half-spherical means. Other hyperplane means which have been studied in relation to subharmonic functions in half-spaces are

$$
\int_{\mathbf{R}^{n}} f(X, y) d X
$$


(see [1] and the papers cited there for a sample of the literature) and

$$
\int_{\mathbf{R}^{n}}\left(1+|X|^{2}\right)^{-\frac{1}{2}(n+1)} f(X, y) d X
$$

$[9,11,12,15]$. An advantage of working with the mean $\Psi_{a}$ is that $\Psi_{a}(f, \cdot)$ is finite on $(a, \infty)$ for a large class of functions $f$, whereas the means (1) and (2) are finite for comparatively small classes of functions. However, in order to obtain interesting conclusions from hypotheses concerning the behaviour of $\Psi_{a}(s, \cdot)$ for a subharmonic function $s$ in $D_{a}$, it is necessary to impose a general restriction on the growth of $s^{+}$. We shall say that a subharmonic function $s$ in $D_{a}$ belongs to the class $\mathscr{S}_{a}$ if for each $b>a$ and each positive number $\lambda$

$$
\lim s^{+}(M) e^{-\lambda|M|}=0
$$

as $M$ tends to the Alexandroff point $\mathscr{A}$ (at infinity) from inside $\Omega_{a, b}$.

We denote the closure and boundary in $\mathbf{R}^{n+1}$ of a set $E$ by $\bar{E}$ and $\partial E$.

Theorem 1. Let s be a non-negative function in $\bar{D}_{a}$ such that $s \in \mathscr{S}_{a}$,

$$
s(N)=\limsup _{\substack{M \rightarrow N \\ M \in D_{a}}} s(M)<\infty \quad\left(N \in \partial D_{a}\right),
$$

and

$$
\int_{\mathbf{R}^{n}}\left(1+|X|^{2}\right)^{-\frac{1}{2}(n+1)} s(X, a) d X<\infty .
$$

Then $\Psi_{a}(s, y)$ is real-valued on $(a, \infty)$ and tends to a limit $\psi_{a}(s)$ as $y \rightarrow \infty$ such that $0 \leqq \psi_{a}(s) \leqq \infty$.

This theorem is of the same type as [10], Theorem 2 and [12], Theorem 2, which deal with the limiting behaviour of half-spherical means and certain weighted hyperplane means, respectively.

Before giving our results on harmonic majorization, we need a brief discussion of Poisson integrals in strips and half-spaces. Let $f$ and $g$ be extended real-valued functions defined on $\mathbf{R}^{n} \times\{a\}$ and $\mathbf{R}^{n} \times\{b\}$, respectively, such that

$$
\int_{\mathbb{R}^{n}}|f(X, a)| e^{-\pi|X| /(b-a)} d X<\infty .
$$

and

$$
\int_{\mathbf{R}^{n}}|g(X, b)| e^{-\pi|X| /(b-a)} d X<\infty .
$$

Then the Poisson integral in $\Omega_{a, b}$ of the function equal to $f$ on $\mathbf{R}^{n} \times\{a\}$ and equal to 0 on $\mathbf{R}^{n} \times\{b\}$ exists and is harmonic in $\Omega_{a, b}$ (see [3], pp. 747,748, 758). We denote this 
Poisson integral by $I_{a, b}$. Similarly, the Poisson integral in $\Omega_{a, b}$ of the function equal to $g$ on $\mathbf{R}^{n} \times\{b\}$ and equal to 0 on $\mathbf{R}^{n} \times\{a\}$ exists and is harmonic in $\Omega_{a, b}$. We denote this Poisson integral by $J_{a, b, g}$. If $F$ is defined on $\partial \Omega_{a, b}$ and if $I_{a, b, F}$ and $J_{a, b, F}$ exist and are harmonic in $\Omega_{a, b}$, we write

$$
H_{a, b, F}=I_{a, b, F}+J_{a, b, F}
$$

Further details of Poisson integrals in strips are given in Sections 2 and 3.

A necessary and sufficient condition for the Poisson integral of $f$ in $D_{a}$ to exist and to be harmonic in $D_{a}$ is

$$
\int_{\mathbf{R}^{n}}|f(X, a)|\left(1+|X|^{2}\right)^{-\frac{1}{2(n+1)}} d X<\infty
$$

(compare [7], Theorem 6). We denote this half-space Poisson integral by $I_{a, \infty, f}$. We shall also need, more generally, half-space Poisson integrals of measures. If $\mu$ is a signed measure on $\mathbf{R}^{n}$ such that

$$
\int_{\mathbf{R}^{n}}\left(1+|X|^{2}\right)^{-\frac{1}{2}(n+1)} d|\mu|(X)<\infty,
$$

then the half-space Poisson integral of $\mu$ in $D_{a}$ is given by

$$
I_{a, \infty, \mu}(M)=\left(2 / s_{n+1}\right) \int_{\mathbf{R}^{n}}(y-a)\left\{|X-Z|^{2}+(y-a)^{2}\right\}^{-\frac{1}{2}(n+1)} d \mu(Z)
$$

and is harmonic in $D_{a}$. Here $s_{n+1}$ is the surface area of the unit sphere in $\mathbf{R}^{n+1}$.

Theorem 2. Let $s$ be a function in $\bar{D}_{a}$ such that $s \in \mathscr{S}_{a}$, (4) holds, and

$$
\int_{\mathbf{R}^{n}}\left(1+|X|^{2}\right)^{-\frac{1}{2(n+1)} s^{+}}(X, a) d X<\infty
$$

Then $\Psi_{a}(s, \cdot)$ and $\Psi_{a}\left(s^{+}, \cdot\right)$ are real-valued on $(a, \infty)$ and $\Psi_{a}\left(s^{+}, y\right)$ tends to a limit $\psi_{a}\left(s^{+}\right)$as $y \rightarrow \infty$ such that $0 \leqq \psi_{a}\left(s^{+}\right) \leqq \infty$.

For $s$ to have a positive harmonic majorant in $D_{a}$ it is necessary and sufficient that $\psi_{a}\left(s^{+}\right)<\infty$.

If $\psi_{0}\left(s^{+}\right)<\infty$, then

(i) $\Psi_{a}(s, y)$ tends to a finite limit $\psi_{a}(s)$ as $y \rightarrow \infty$,

(ii) $\int_{\mathbf{R}^{n}}\left(1+|X|^{2}\right)^{-\frac{1}{2}(n+1)}|s(X, a)| d X<\infty$,

(iii) $\lim _{b \rightarrow \infty} I_{a, b, s}(M)=I_{a, \infty, s}(M) \quad\left(M \in D_{a}\right)$,

(iv) $\lim _{b \rightarrow \infty} J_{a, b, s}(M)=\left(c_{n}\right)^{-1} \psi_{a}(s)(y-a) \quad\left(M \in D_{a}\right)$,

where

$$
c_{n}=\int_{\mathbb{R}^{n}}(1+|Z|)^{ \pm(1-n)} e^{-\pi|Z|} d Z
$$


(v) the function $h_{s, a}$, defined in $D_{a}$ by writing

$$
h_{s, a}(M)=I_{a, \infty, s}(M)+\left(c_{n}\right)^{-1} \psi_{s}(s)(y-a),
$$

is a harmonic majorant of $s$ in $D_{a}$.

Corollary. If $s \in \mathscr{S}_{a}$ and

$$
\limsup _{\substack{M \rightarrow N \\ M \in D_{a}}} s(M) \leqq 0 \quad\left(N \in \partial D_{a}\right)
$$

and $\psi_{a}\left(s^{+}\right)=0$, then $s \leqq 0$, in $D_{a}$.

Under the hypotheses of Theorem 2 it is possible that the function $h_{s, a}$, defined by (11), is a harmonic majorant of $s$ in $D_{a}$ but is not the least such majorant. However, the following theorem gives sufficient conditions for $h_{s, a}$ to be the least harmonic majorant of $s$ in $D_{a}$.

Theorem 3. Suppose that $a>0$ and that $s \in \mathscr{S}_{0}$. Then $s$ has a positive harmonic majorant in $D_{a}$ if and only if $(10)$ holds and $\psi_{a}\left(s^{+}\right)<\infty$. Further, if these conditions are satisfied, then the least harmonic majorant of $s$ in $D_{a}$ is the function $h_{s, a}$ given by (11).

The example $s(M)=-\sqrt{ }(y-a)$ shows that the conditions in Theorem 3 are not necessary for its final conclusion; this function is subharmonic in $D_{a}$ but has no subharmonic extension to $D_{0}$.

Finally, we consider $\psi_{a}(s)$ as a function of $a$.

Theorem 4. Let $s$ be defined in $\bar{D}_{a}$. If $s \in \mathscr{S}_{a}$ and $s$ satisfies (4) and (10) and if $\psi_{a}\left(s^{+}\right)<\infty$, then $\psi .(s)$ is constant on $[a, \infty)$.

A similar result for half-spherical means is given in [12], Theorem 1.

\section{Preliminaries on Poisson integrals in strips}

We recapitulate some of Brawn's results.

Let $\Phi:[0, \infty) \times(0,2) \rightarrow \mathbf{R}$ be defined by

$$
\begin{gathered}
\Phi(0, y)=(2 \pi)^{-\frac{1}{2} n} 2^{1-\frac{1}{2} n}\left\{\Gamma\left(\frac{1}{2} n\right)\right\}^{-1} \int_{0}^{\infty} t^{n-1} \sinh \{(1-y) t\}(\sinh t)^{-1} d t \\
\Phi(r, y)=(2 \pi)^{-\frac{1}{2} n} \int_{0}^{\infty} t^{\frac{1}{2} n} r^{1-\frac{1}{2} n} J_{\frac{1}{2} n-1}(r t) \sinh \{(1-y) t\}(\sinh t)^{-1} d t \quad(r>0),
\end{gathered}
$$

where $J_{\frac{1}{2} n-1}$ denotes the Bessel function of the first kind of order $\frac{1}{2} n-1$ ([14], p. 40). Then $\Phi$ is positive and continuous on $[0, \infty) \times(0,1)$. If $f$ and $g$ are functions satisfying 
(6) and (7), then $I_{a, b, f}$ and $J_{a, b, g}$ are given by

$$
I_{a, b, f}(M)=(b-a)^{-n} \int_{\mathbf{R}^{n}} \Phi(|X-Z| /(b-a),(y-a) /(b-a)) f(Z, a) d Z
$$

and

$$
J_{a, b, g}(M)=(b-a)^{-n} \int_{\mathbf{R}^{n}} \Phi(|X-Z| /(b-a),(b-y) /(b-a)) g(Z, b) d Z .
$$

Lemma A. If $f$ is a function on $\mathbf{R}^{n} \times\{a\}$ satisfying (6) then $I_{a, b, f}$ is harmonic in $\Omega_{a, b}$ and

$$
\lim _{M \rightarrow N} I_{a, b, f}(M)=0 \quad\left(N \in \mathbf{R}^{n} \times\{b\}\right)
$$

If, further, $f$ is continuous at a point $P$ of $\mathbf{R}^{n} \times\{a\}$, then

$$
\lim _{M \rightarrow P} I_{a, b, f}(M)=f(P)
$$

If $f$ has compact support, then

$$
\lim _{\mathscr{M} \rightarrow \infty} I_{a, b, f}(M)=0
$$

The same results, with $\mathbf{R}^{n} \times\{a\}$ and $\mathbf{R}^{n} \times\{b\}$ interchanged, hold for $J_{a, b, g}$, where $g$ is $a$ function on $\mathbf{R}^{n} \times\{b\}$ satisfying (7).

The results for $I_{a, b, f}$ are contained in [3] (Theorem 1, Lemmas 1,2) in the case where $a=0$ and $b=1$. For an indication of the modifications required to pass to the general case, see [3], p. 758. It is easy to see that the corresponding results hold for $J_{a, b, g}$.

Next, we give the results on harmonic majorization in strips that we shall need.

Lemma B. If $s$ is defined in $\Omega_{a, b}$ and is subharmonic in $\Omega_{a, b}$ and satisfies

$$
\begin{aligned}
& \limsup _{\substack{M \rightarrow N \\
M \in \mathbf{\Omega}_{a, b}}} s(M)=s(N)<\infty \quad\left(N \in \partial \Omega_{a, b}\right), \\
& \int_{\mathbf{R}^{n}}\{|s(Z, a)|+|s(Z, b)|\} e^{-\pi|Z| /(b-a)} d Z<\infty
\end{aligned}
$$

and

$$
\lim _{\substack{M \rightarrow \infty \\ M \in \mathbf{\Omega}_{a, b}^{-\infty}}} s^{+}(M) e^{-\pi|X| /(b-a)}|X|^{\frac{1}{2}(n-1)}=0,
$$

then $H_{a, b, s}$ is a harmonic majorant of $s$ in $\Omega_{a, b}$.

Lemma C. If $0 \leqq \alpha<a<b<\beta$ and $s$ is subharmonic in $\Omega_{\alpha, \beta}$ and has a positive harmonic majorant there, then the least harmonic majorant of $s$ in $\Omega_{a, b}$ is $H_{a, b, s}$. 
In the case where $a=0$ and $b=1$ Lemma $B$ is [3], Theorem 2, and in the case where $\alpha=0$ and $\beta=1$ Lemma $C$ is [4], Theorem 2. The stated generalizations are easily obtained from the cited cases.

\section{Further results on Poisson integrals in strips}

We use $A$ to denote a finite positive constant depending at most on $n$, not necessarily the same on any two occurrences.

Lemma 1. If $0 \leqq a<c \leqq \frac{1}{2}(a+b)$ and if $g$ is a non-negative function on $\mathbf{R}^{n} \times\{b\}$ such that

$$
\int_{\mathbf{R}^{n}} g(X, b) e^{-\pi|X| /(b-a)} d X<\infty
$$

then

$$
A J_{a, b, g}(0, \ldots, 0, c) \leqq(c-a) \Psi_{a}(g, b) \leqq A J_{a, b, g}(0, \ldots, 0, c)
$$

We start by showing that

$$
A \sin (\pi y)(1+r)^{\frac{1}{2}(1-n)} e^{-\pi r} \leqq \Phi(r, y) \leqq A \sin (\pi y)(1+r)^{\frac{1}{2}(1-n)} e^{-\pi r}
$$

whenever $r \geqq 0$ and $\frac{1}{2}<y<1$. A similar but slightly less general result than (12) is given in [4], Lemma 1. Our proof of (12) for large $r$ is modelled on the proof in [4]. We start from the equation

$$
\Phi(r, y)=(2 r)^{1-\frac{1}{2} n} \sum_{m=1}^{\infty} m^{\frac{1}{2} n} \sin (m \pi y) K_{\frac{1}{n} n-1}(m \pi r) \quad(r>0,0<y<1),
$$

where $K_{t n-1}$ denotes the Bessel function of the third kind of order $\frac{1}{2} n-1$ ([14], p. 78). For this equation, see [2], formula (22) and note that $\Phi$ is normalized in accordance with [4] and not [2]. Hence when $r \geqq 1$ and $0<y<1$

$$
\begin{aligned}
& \left|\Phi(r, y)-(2 r)^{1-\frac{1}{2} n} \sin (\pi y) K_{\frac{1}{2} n-1}(\pi r)\right| \\
& =(2 r)^{1-\frac{1}{2} n}\left|\sum_{m=2}^{\infty} m^{\frac{1}{2} n} \sin (m \pi y) K_{\frac{1}{2} n-1}(m \pi r)\right| \\
& \leqq(2 r)^{1-\frac{1}{2} n} \sin (\pi y) \sum_{m=2}^{\infty} m^{\frac{1}{2 n+1}} K_{\frac{1}{2} n-1}(m \pi r) \\
& \leqq A r^{\frac{t}{2(1-n)}} \sin (\pi y) \sum_{m=2}^{\infty} m^{\frac{1}{2(n+1)}} e^{-m \pi r} \\
& \leqq A r^{\frac{1}{t}(1-n)} \sin (\pi y) e^{-2 \pi r} \sum_{m=2}^{\infty} m^{\frac{1}{2(n+1)}} e^{-(m-2)} \\
& =A r^{\frac{1}{2(1-n)}} \sin (\pi y) e^{-2 \pi r} \text {. }
\end{aligned}
$$


The first of the above inequalities follows from the inequalities

$$
K_{\frac{1}{2} n-1}(\xi)>0 \quad(\xi>0) \quad|\sin (m \pi y)| \leqq m \sin (\pi y) \quad(0<y<1, m=1,2, \ldots),
$$

and the second follows from the inequality

$$
K_{\frac{\ddagger}{\ddagger} n-1}(\xi) \leqq A \xi^{-\frac{1}{2}} e^{-\xi} \quad(\xi \geqq 1) \quad([14], \text { p. } 219) .
$$

Since as $r \rightarrow \infty$

$$
K_{\frac{1}{2} n-1}(\pi r)=(2 r)^{-\frac{1}{2}} e^{-\pi r}(1+o(1)) \quad([14], \text { p. } 219),
$$

it follows from (13) that

$$
\begin{aligned}
\Phi(r, y) & =(2 r)^{\frac{1}{(1}(1-n)} \sin (\pi y) e^{-\pi r}(1+o(1)) \\
& =2^{\frac{1}{2}(1-n)} \sin (\pi y)(1+r)^{\frac{1}{t^{(1-n)}}} e^{-\pi r}(1+o(1)) .
\end{aligned}
$$

Hence (12) holds when $r$ is larger than some positive number $r_{0}=r_{0}(n)$ and $0<y<1$.

Now define a function $h_{1}$ in $\Omega_{0,2}$ by writing

$$
h_{1}(X, y)=\Phi(|X|, y) \text {. }
$$

Then $h_{1}$ is harmonic in $\Omega_{0,2}$ and vanishes on $\mathbf{R}^{n} \times\{1\}$. (It is the Poisson kernel of $\Omega_{0,1}$ with pole at the origin, see [2]). Hence $\left|\partial h_{1} / \partial y\right| \leqq A$ in the set $\left\{(X, y):|X| \leqq r_{0}, \frac{1}{2} \leqq y \leqq 1\right\}$ and therefore if $0 \leqq r \leqq r_{0}$ and $\frac{1}{2} \leqq y<1$, then by the mean value theorem, there exists $y^{\prime} \in(y, 1)$ such that

$$
\begin{aligned}
|\Phi(r, y)| & =\left|h_{1}(r, 0, \ldots, 0, y)-h_{1}(r, 0, \ldots, 0,1)\right| \\
& =(1-y)\left|\frac{\partial h}{\partial y}\left(r, 0, \ldots, 0, y^{\prime}\right)\right| \\
& \leqq A(1-y) \leqq A \sin (\pi y),
\end{aligned}
$$

and it now follows that the right-hand inequality in (12) holds whenever $r \geqq 0$ and $\frac{1}{2} \leqq y<1$.

Next define $h_{2}$ in $\mathbf{R}^{n+1}$ by writing

$$
h_{2}(X, y)=\cos \left(\pi x_{1} / 4 r_{0}\right) \ldots \cos \left(\pi x_{n} / 4 r_{0}\right) \sinh \left(\pi \sqrt{ } n(1-y) / 4 r_{0}\right) .
$$

It is easy to check that $h_{2}$ is harmonic in $\mathbf{R}^{n+1}$. Further, if

$$
\omega=\left\{(X, y):\left|x_{i}\right|<2 r_{0}(i=1, \ldots, n), \quad \frac{1}{2}<y<1\right\},
$$


then $h_{1}>A$ and $h_{2} \leqq \sinh \left(\pi \sqrt{ } n / 8 r_{0}\right)$ on $\partial \omega \cap \partial D_{\frac{1}{2}}$ and $h_{1} \geqq 0=h_{2}$ on $\partial \omega \cap D_{\frac{1}{2}}$. Hence $h_{1} \geqq A h_{2}$ on $\partial \omega$, and it follows from the minimum principle that $h_{1} \geqq A h_{2}$ in $\bar{\omega}$. Hence if $0 \leqq r \leqq r_{0}$ and $\frac{1}{2} \leqq y \leqq 1$, then

$$
\begin{aligned}
\Phi(r, y) & =h_{1}(r, 0, \ldots, 0, y) \geqq A h_{2}(r, 0, \ldots, 0, y) \\
& \geqq 2^{-\frac{1}{2}} \sinh \left(\pi \sqrt{ } n(1-y) / 4 r_{0}\right) \\
& \geqq 2^{-5 / 2}\left(r_{0}\right)^{-1} \pi \sqrt{ } n(1-y) \geqq A \sin (\pi y)
\end{aligned}
$$

It now follows that the left-hand inequality in (12) holds whenever $r \geqq 0$ and $\frac{1}{2} \leqq y \leqq 1$, and the proof of (12) is complete.

If $a, b$ and $c$ are as in the lemma, then $\frac{1}{2} \leqq(b-c) /(b-a)<1$. Hence, by (12), for each $Z \in \mathbf{R}^{n}$

$$
\Phi(|Z| /(b-a),(b-c) /(b-a))
$$

lies between positive multiples of

$$
\sin \{\pi(b-c) /(b-a)\}\{1+|Z| /(b-a)\}^{\frac{1}{2}(1-n)} e^{-\pi|Z| /(b-a)}
$$

(the implied constants depending only on $n$ ). Since, for such $a, b$ and $c$,

$$
2(c-a) /(b-a)<\sin \{\pi(b-c) /(b-a)\}<\pi(c-a) /(b-a),
$$

it follows that (14) lies between positive multiples of

$$
(c-a)(b-a)^{-1}\{1+|Z| /(b-a)\}^{\frac{1}{2}(1-n)} e^{-\pi|Z| /(b-a)}
$$

(the implied constants again depending only on $n$ ). Hence the lemma follows.

We need some results on the Perron-Wiener-Brelot (PWB) solution of the Dirichlet problem (see, for example, [8] for a general account). If $\Omega$ is an unbounded domain in $\mathbf{R}^{n+1}$, we denote its compactified boundary $\partial \Omega \cup\{\mathscr{A}\}$ by $\partial^{*} \Omega$. A function $F$, defined at least on $\partial^{*} \Omega$, such that the PWB solution of the Dirichlet problem in $\Omega$ with boundary data $F$ exists and is harmonic in $\Omega$ is called resolutive, and we denote the PWB solution by $H(\Omega, F)$.

Lemma 2. Let $f$ and $g$ be functions on $\mathbf{R}^{n} \times\{a\}$ and $\mathbf{R}^{n} \times\{b\}$ respectively.

(i) Define $F_{1}$ on $\partial^{*} \Omega_{a, b}$ by writing

$$
F_{1}(M)=f(M)\left(M \in \mathbf{R}^{n} \times\{a\}\right), \quad F_{1}(M)=g(M)\left(M \in \mathbf{R}^{n} \times\{b\}\right), \quad F_{1}(\mathscr{A})=0
$$

If $f$ and $g$ satisfy (6) and (7), then $F_{1}$ is resolutive and $H\left(\Omega_{a, b}, F_{1}\right)=I_{a, b, f}+J_{a, b, g}$ in $\Omega_{a, b}$. 
(ii) Define $F_{2}$ on $\partial^{*} D_{a}$ by writing

$$
F_{2}(M)=f(M) \quad\left(M \in \partial D_{a}\right), \quad F_{2}(\mathscr{A})=0
$$

Then $F_{2}$ is resolutive if and only if (8) holds, and in this case $H\left(D_{a}, F_{2}\right)=I_{a, \infty, f}$.

We prove only (i), the proof of (ii) being similar. If $f$ and $g$ are real-valued and continuous in their domains of definition and have compact supports, then $I_{a, b, f}+J_{a, b, g}$ is harmonic in $\Omega_{a, b}$ and by Lemma $A$,

$$
\lim _{M \rightarrow N}\left\{I_{a, b, f}(M)+J_{a, b, g}(M)\right\}=F_{1}(N) \quad\left(N \in \partial^{*} \Omega_{a, b}\right)
$$

It follows that $I_{a, b, f}+J_{a, b, g}$ is the classical solution and hence the PWB solution of the Dirichlet problem in $\Omega_{a, b}$ with boundary data $F_{1}$. It follows from this special case that the harmonic measure on $\partial^{*} \Omega_{a, b}$ relative to a point $(X, y)$ of $\Omega_{a, b}$ is given on $\mathbf{R}^{n} \times\{a\}$ by

$$
(b-a)^{-n} \Phi(|X-Z| /(b-a),(y-a) /(b-a)) d Z
$$

and on $\mathbf{R}^{n} \times\{b\}$ by

$$
(b-a)^{-n} \Phi(|X-Z| /(b-a),(b-y) /(b-a)) d Z,
$$

whence the general result follows.

\section{Means of half-space Poisson integrals and potentials}

Lemma 3. Let $\mu$ be a signed measure on $\mathbf{R}^{\boldsymbol{n}}$ such that (9) holds. Then $\Psi_{a}\left(I_{a, \infty, \mu}, y\right)$ is finite on $(a, \infty)$ and tends to 0 as $y \rightarrow \infty$.

We may suppose, without loss of generality, that $a=0$. Then

$$
\begin{aligned}
& \frac{1}{2} S_{n+1}\left|\Psi_{0}\left(I_{0, \infty, \mu}, y\right)\right| \\
& \quad=y^{-n-1}\left|\int_{\mathbf{R}^{n}}(1+|X| / y)^{\frac{1}{2}(1-n)} e^{-\pi|X| / y} \int_{\mathbf{R}^{n}} y\left(y^{2}+|X-Z|^{2}\right)^{-\frac{1}{2}(n+1)} d \mu(Z) d X\right| \\
& \quad \leqq y^{-n} \int_{\mathbf{R}^{n}} \int_{\mathbf{R}^{n}}(1+|X| / y)^{\frac{1}{2}(1-n)}\left(y^{2}+|X-Z|^{2}\right)^{-\frac{1}{2}(n+1)} e^{-\pi|X| / y} d X d|\mu|(Z) \\
& \quad \leqq y^{-n} \int_{\mathbf{R}^{n}} \int_{\mathbf{R}^{n}}\left(y^{2}+|X-Z|^{2}\right)^{-\frac{1}{2}(n+1)} e^{-\pi|X| / y} d X d|\mu|(Z) .
\end{aligned}
$$


Now, for each $Z \in \mathbf{R}^{n}$, putting $X=y X^{\prime}$ and $Z=y Z^{\prime}$, we have

$$
\begin{aligned}
\int_{\mathbf{R}^{n}}\left(y^{2}\right. & \left.+|X-Z|^{2}\right)^{-\frac{1}{2}(n+1)} e^{-\pi|X| / y} d X \\
& =y^{-1} \int_{\mathbf{R}^{n}}\left(1+\left|X^{\prime}-Z^{\prime}\right|^{2}\right)^{-\frac{1}{2}(n+1)} e^{-\pi\left|X^{\prime}\right|} d X^{\prime} \\
& \leqq A y^{-1} \int_{\mathbf{R}^{n}}\left\{\left(1+\left|X^{\prime}-Z^{\prime}\right|^{2}\right)\left(1+\left|X^{\prime}\right|^{2}\right)\right\}^{-\frac{1}{2}(n+1)} d X^{\prime} \\
& =A y^{-1}\left(4+\left|Z^{\prime}\right|^{2}\right)^{-\frac{1}{2}(n+1)} \\
& =A y^{n}\left(y^{2}+|Z|^{2}\right)^{-\frac{1}{2}(n+1)} .
\end{aligned}
$$

To prove the last written equality, note that the integral in (16) is a constant positive multiple of the value at $\left(Z^{\prime}, 1\right)$ of the Poisson integral in $D_{0}$ of the function $\left(1+\left|X^{\prime}\right|^{2}\right)^{-\frac{1}{2}(n+1)}$ and use the reproductive property of the Poisson kernel. From (15) and (17) we obtain

$$
\left|\Psi_{0}\left(I_{0, \infty, \mu}, y\right)\right| \leqq A \int_{\mathbf{R}^{n}}\left(y^{2}+|Z|^{2}\right)^{-\frac{1}{2}(n+1)} d|\mu|(Z) .
$$

Since $\mu$ satisfies (9), the right-hand side of (18) is finite for each positive $y$ and tends to 0 as $y \rightarrow \infty$, by Lebesgue's dominated convergence theorem.

Recall that a superharmonic function in a domain $\Omega$ is called a potential if its greatest harmonic minorant in $\Omega$ is identially zero.

Lemma 4. If $u$ is a potential in $D_{a}$, then $\Psi_{a}(u, y)$ is finite on $(a, \infty)$ and tends to 0 as $y \rightarrow \infty$.

Again it suffices to work with $a=0$. In [12], Theorem 3 it was shown that if $u$ is a potential in $D_{0}$, then the function

$$
K(u, y)=\int_{\mathbf{R}^{n}}\left\{|X|^{2}+(y+1)^{2}\right\}^{-\frac{1}{2}(n+1)} u(X, y) d X
$$

is real-valued for $y>0$ and tends to 0 as $y \rightarrow \infty$. We use this result to prove Lemma 4 . Suppose that $y_{0}>0$ and that $(X, y) \in D_{y_{0}}$. Then

$$
|X|^{2}+(y+1)^{2} \leqq C(y+|X|)^{2},
$$

where $C$ depends only on $y_{0}$. Hence

$$
\begin{gathered}
y^{-n-1}(1+|X| / y)^{\frac{1}{2(1-n)}} e^{-\pi|X| / y}\left\{|X|^{2}+(y+1)^{2}\right\}^{\frac{1}{1(n+1)}} \\
\leqq C^{\frac{1}{2(n+1)}}(1+|X| / y)^{\frac{1}{2}(n+3)} e^{-\pi|X| / y}
\end{gathered}
$$

which is bounded on $D_{y_{0}}$. It now follows that $\Psi_{0}(u, \cdot)$ is dominated by a constant multiple of $K(u, \cdot)$ and, in view of the properties of $K(u, \cdot)$, this proves the lemma. 
Lemma 5. If

$$
v(M)=y-a \quad\left(M \in D_{a}\right)
$$

then $\Psi_{a}(v, \cdot) \equiv c_{n}$ on $(a, \infty)$.

This is the result of a simple calculation which we omit.

\section{Proof of Theorem 1}

The following lemmas will be useful in the proofs of Theorems 1 and 2 .

Lemma 6. If $f$ is a function on $\partial D_{a}$ which satisfies (8), then for each $M \in D_{a}$

$$
\lim _{b \rightarrow \infty} I_{a, b, f}(M)=I_{a, \infty, f}(M)
$$

If, further, $f \geqq 0$ on $\partial D_{a}$, then for each $b \in(a, \infty)$, we have $I_{a, b, f} \leqq I_{a, \infty, f}$ in $\Omega_{a, b}$.

Lemma 7. If s satisfies the hypotheses of Theorem 1, then for each $M=(X, y) \in D_{a}$, we have that $H_{a, b, s}(M)$ is increasing (in the wide sense) as a function of $b$ on $(y, \infty)$.

The proof of Lemma 6 depends on the following result.

Lemma D. Let $\Omega_{0}$ and $\Omega$ be unbounded domains in $\mathbf{R}^{n+1}$ such that $\Omega \subset \Omega_{0}$. Let $F$ be a function on $\Omega_{0} \cup \partial^{*} \Omega_{0}$ such that $F$ is resolutive on $\partial^{*} \Omega_{0}$ and $F=H\left(\Omega_{0}, F\right)$ in $\Omega_{0}$. Then $F$ is resolutive on $\partial^{*} \Omega$ and $F=H(\Omega, F)$ in $\Omega$.

See [5], p. 98, for the corresponding result in bounded domains.

To prove Lemma 6 , define $F$ in $D_{a} \cup \partial^{*} D_{a}$ by putting

$$
F(M)=I_{a, \infty, f}(M) \quad\left(M \in D_{a}\right), \quad F(M)=f(M) \quad\left(M \in \partial D_{a}\right), \quad F(\mathscr{A})=0 .
$$

Then, by Lemma 2(ii), $F$ is resolutive on $\partial^{*} D_{a}$ and $F=H\left(D_{a}, F\right)$ in $D_{a}$. Hence, by Lemma $\mathrm{D}, F$ is resolutive on $\partial^{*} \Omega_{a, b}$ and $F=H\left(\Omega_{a, b}, F\right)$ in $\Omega_{a, b}$. By Lemma 2(i), we also have in $\Omega_{a, b}$

$$
H\left(\Omega_{a, b}, F\right)=H_{a, b, F}=I_{a, b, f}+J_{a, b, F} .
$$

Hence

$$
I_{a, \infty, f}=I_{a, b, f}+J_{a, b, F}
$$

in $\Omega_{a, b}$. If $f \geqq 0$ on $\partial D_{a}$, then $F \geqq 0$ in $D_{a}$ and $J_{a, b, F} \geqq 0$ in $\Omega_{a, b}$, so the inequality stated in the lemma now follows. To prove (19), it now suffices to show that $J_{a, b, F}(M) \rightarrow 0$ as $b \rightarrow \infty$ for each $M \in D_{a}$. Since $F$ is a half-space Poisson integral in $D_{a}$, we have by 
Lemma $3, \Psi_{a}(F, b) \rightarrow 0$ as $b \rightarrow \infty$. From Lemma 1 it now follows, in the case where $f \geqq 0$ on $\partial D_{a}$ that

$$
\lim _{b \rightarrow \infty} J_{a, b, F}(0, \ldots, 0, y)=0
$$

for each $y>a$. In the case where $f$ takes values of both signs, the same conclusion follows by working with $f^{+}$and $f^{-}$. Since we may translate the origin parallel to the $x_{1}, \ldots, x_{n}$-axes, we find that $J_{a, b, F}(M) \rightarrow 0$ as $b \rightarrow \infty$ for each $M \in D_{a}$, as required.

To prove Lemma 7, suppose that $a<b<b^{\prime}$ and define $w$ in $\bar{D}_{a}$ to be equal to $H_{a, b, s}$ in $\Omega_{a, b}$ and equal to $s$ elsewhere in $\bar{D}_{a}$. Then $w \geqq s$ in $\Omega_{a, b}$ ([3], Theorem 2 , interpreted for $\left.\boldsymbol{\Omega}_{a, b}\right)$ and $w$ is subharmonic in $D_{a}([4]$, p. 280). It is easy to check that $w$ satisfies the conditions of [3], Theorem 2, interpreted for $\Omega_{a, b^{\prime}}$. Hence $H_{a, b^{\prime}, s} \geqq w=H_{a, b, s}$ in $\Omega_{a, b}$.

Lemma E. If $s$ is subharmonic in $D_{a}$ and $s$ has a positive harmonic majorant in $D_{a}$, then $s$ is expressible in the form

$$
s(M)=I_{a, \infty, \mu}(M)+k(y-a)-u(M) \quad\left(M \in D_{a}\right),
$$

where $\mu$ is a signed measure on $\mathbf{R}^{n}$ satisfying (9), $k$ is a real number and $u$ is a potential in $D_{a}$.

This result is essentially known. It can be deduced from [12], Theorem 5(ii) and the Riesz decomposition theorem in the form given, for example, in [12], Theorem $\mathrm{C}$.

We can now complete the proof of Theorem 1 . Since $s \in \mathscr{S}_{a}$, it is clear that $\Psi_{a}(s, \cdot)$ is finite on $(a, \infty)$. Since, by Lemma $7, H_{a, b, s}$ is an increasing function of $b$ in $D_{a}$, and since, by Lemma $6, I_{a, b, s} \rightarrow I_{a, \infty, s}$ in $D_{a}$ as $b \rightarrow \infty$, it follows that either $J_{a, b, s} \rightarrow \infty$ in $D_{a}$ or $J_{a, b, s}$ tends to a harmonic limit in $D_{a}$ as $b \rightarrow \infty$. In the former case, it follows from Lemma 1 that $\Psi_{a}(s, b) \rightarrow \infty$ as $b \rightarrow \infty$. In the latter case, $s$ has a harmonic majorant in $D_{a}$, since, by Lemma $\mathrm{B}, H_{a, b, s} \geqq s$ in $\Omega_{a, b}$ and since $\lim _{b \rightarrow \infty} H_{a, b, s}$ is harmonic in $D_{a}$. Hence, in this case, by Lemma E, $s$ has the representation (20) in $D_{a}$, so that

$$
\begin{gathered}
\Psi_{a}(s, y)=\Psi_{a}\left(I_{a, \infty, \mu}, y\right)+k \Psi_{a}(y-a, y)-\Psi_{a}(u, y) \\
\rightarrow 0+c_{m} k-0 \quad(y \rightarrow \infty),
\end{gathered}
$$

by Lemmas 3, 4 and 5 .

\section{Proof of Theorem 2}

Clearly, if $s$ satisfies the hypotheses of Theorem 2 , then $s^{+}$satisfies the hypotheses of Theorem 1 , so that $\Psi_{a}\left(s^{+}, y\right)$ is finite on $(a, \infty)$ and tends to a limit $\psi_{a}\left(s^{+}\right)$as $y \rightarrow \infty$ such that $0 \leqq \psi_{a}\left(s^{+}\right) \leqq \infty$.

For each positive integer $m$, define $s_{m}$ in $\bar{\Omega}_{a, b}$ to be $\max \{-m, s\}$. Then each $s_{m}$ satisfies the hypotheses of Lemma B, so that $H_{a, b, s_{m}}$ is a harmonic majorant of $s_{m}$ in $\Omega_{a, b}$. By monotone convergence, $H_{a, b, s_{m}} \rightarrow H_{a, b, s}$ in $\Omega_{a, b}$ as $m \rightarrow \infty$. Hence $H_{a, b, s}$ is a harmonic 
majorant of $s$ in $\Omega_{a, b}$. In particular, this implies that

$$
J_{a, b, s}\left(0, \ldots, 0, \frac{1}{2}(a+b)\right)>-\infty
$$

so that

$$
J_{a, b, s}-\left(0, \ldots, 0, \frac{1}{2}(a+b)\right)<\infty \text {. }
$$

Hence, by Lemma $1, \Psi_{a}\left(s^{-}, b\right)<\infty$, and since $\Psi_{a}\left(s^{+}, b\right)<\infty$, it now follows that $\Psi_{a}(s, \cdot)$ is finite on $(a, \infty)$.

Now suppose that $s$ has a positive harmonic majorant in $D_{a}$. Then

$$
\int_{\mathbf{R}^{n}}\left(|X|^{2}+y^{2}\right)^{-\frac{1}{2}(n+1)} s^{+}(X, y) d X
$$

is bounded on $(a+1, \infty)$ ([9], Theorem 4) and since $\Psi_{a}\left(s^{+}, y\right)$ is dominated by a positive multiple of this integral for $y>a+1$ (cf. proof of Lemma 4 above), we have $\Psi_{a}\left(s^{+}\right)<\infty$.

Conversely, suppose that $\psi_{a}\left(s^{+}\right)<\infty$. By Lemmas B and $7, H_{a, b, s^{+}}$is a harmonic majorant of $s^{+}$in $\Omega_{a, b}$ and increases with $b$. Hence it follows easily that as $b \rightarrow \infty$, either $H_{a, b, s^{+}} \rightarrow \infty$ in $D_{a}$ or $H_{a, b, s^{+}}$tends to a limit function which is a harmonic majorant of $s^{+}$in $D_{a}$. To show that $s$ has a positive harmonic majorant in $D_{a}$, it now suffices to prove that for some $M \in D_{a}$

$$
\lim _{b \rightarrow \infty} H_{a, b, s^{+}}(M)<\infty
$$

By Lemma 1 , if $b \geqq a+2$, then

$$
J_{a, b, s^{+}}(0, \ldots, 0, a+1) \leqq A \Psi_{a}\left(s^{+}, b\right),
$$

so that

$$
\lim _{b \rightarrow \infty} \sup J_{a, b, s^{+}}(0, \ldots, 0, a+1)<\infty
$$

and by Lemma 6,

$$
I_{a, b, s^{+}}(0, \ldots, 0, a+1) \leqq I_{a, \infty, s^{+}}(0, \ldots, 0, a+1)<\infty .
$$

Hence (21) holds with $M=(0, \ldots, 0, a+1)$.

For the remainder of this section we suppose that $\psi_{a}\left(s^{+}\right)<\infty$ and we show that (i)(v) hold.

Since $s$ has a positive harmonic majorant in $D_{a}$, by Lemma $\mathrm{E}$, we can write $s$ in the form (20), so that, by Lemmas 3, 4 and 5

$$
\lim _{y \rightarrow \infty} \psi_{a}(s, y)=c_{n} k
$$


To prove (ii), note that

$$
\int_{\mathbf{R}^{n}}\left\{(y+1-a)^{2}+|X|^{2}\right\}^{-\frac{1}{2}(n+1)} s^{-}(X, y) d X
$$

is bounded for $y \in(a, \infty)$, by [12], Theorem 5(i), interpreted for $D_{a}$. Since $s^{-}$is lower semi-continuous in $\bar{D}_{a}$, on letting $y \rightarrow a^{+}$, we obtain, by Fatou's lemma,

$$
\int_{\mathbf{R}^{n}}\left(1+|X|^{2}\right)^{-\frac{1}{2}(n+1)} s^{-}(X, a) d X<\infty,
$$

and this, with (10), gives the result.

Conclusion (iii) now. follows from Lemma 6.

To prove (iv), we use again the representation (20) of $s$. Writing $H=I_{a, \infty, \mu}$, we have $J_{a, b, H} \rightarrow 0$ in $D_{a}$ as $b \rightarrow \infty$ (cf. proof of Lemma 6). Also, by Lemmas 1 and 4, if $y>a$, then

$$
\begin{aligned}
0 & \leqq \lim _{b \rightarrow \infty} J_{a, b, u}(0, \ldots, 0, y) \\
& \leqq A(y-a) \lim _{b \rightarrow \infty} \Psi_{a}(u, b)=0 .
\end{aligned}
$$

Since we may translate the origin parallel to the $x_{1}, \ldots, x_{n}$-axes, we find that $J_{a, b, u} \rightarrow 0$ in $D_{a}$ as $b \rightarrow \infty$. It now follows that

$$
\lim _{b \rightarrow \infty} J_{a, b, s}(M)=k \lim _{b \rightarrow \infty} J_{a, b, y-a}(M) \quad\left(M \in D_{a}\right)
$$

From Lemma 2(i) it is easy to see that $J_{a, b, y-a}(M)=y-a$ when $M \in \Omega_{a, b}$. Hence

$$
\lim _{b \rightarrow \infty} J_{a, b, s}(M)=k(y-a) \quad\left(M \in D_{a}\right)
$$

and since $\psi_{a}(s)=c_{n} k$ (see (22)), the result follows.

The conclusion (v) now follows from (iii) and (iv), since, by Lemma B, $H_{a, b, s}$ is a harmonic majorant of $s$ in $\Omega_{a, b}$.

To prove the corollary, first extend $s$ to $\bar{D}_{a}$ by writing

$$
s(N)=\limsup _{\substack{M \rightarrow N \\ M \in D_{a}}} s(M) \quad\left(N \in \partial D_{a}\right) .
$$

Thus extended, $s$ satisfies the hypotheses of Theorem 2, and therefore the function $h_{s, a}$, given by (11), is a harmonic majorant of $s$ in $D_{a}$. Since $s \leqq 0$ on $\partial D_{a}$, we have $I_{a, \infty, s} \leqq 0$ in $D_{a}$. Since, also, $\psi_{a}(s) \leqq \psi_{a}\left(s^{+}\right)=0$, it follows that $h_{s, a} \leqq 0$ in $D_{a}$. Hence $s \leqq 0$ in $D_{a}$.

\section{Proof of Theorem 3}

If (10) holds and if $\psi_{a}\left(s^{+}\right)<\infty$, then it follows from Theorem 2 that $s$ has a positive harmonic majorant in $D_{a}$. 
Conversely, if $s$ has such a majorant, then $s^{+}$has a harmonic majorant in $D_{a}$ and (10) holds, by [9], Theorem 3 and $\psi_{a}\left(s^{+}\right)<\infty$ by Theorem 2 .

To prove the last assertion in the theorem, suppose that $0<\alpha<a<b<\beta<\gamma$ and define $h$ in $\mathbf{R}^{n+1}$ by

$$
h(X, y)=\cosh \left(x_{1} \pi / \gamma \sqrt{ } n\right) \ldots \cosh \left(x_{n} \pi / \gamma \sqrt{ } n\right) \sin (y \pi / \gamma)
$$

It is easy to check that $h$ is harmonic in $\mathbf{R}^{n+1}$. Also, $h(M) \geqq e^{C|M|}$ when $M \in \Omega_{\alpha, \beta}$, where $C$ is a positive constant depending only on $\alpha, \beta, \gamma$ and $n$. Since $s \in \mathscr{S}_{0}$, it is clear that $s$ is majorized in $\Omega_{\alpha, \beta}$ by some multiple of $h$. Hence, by Lemma $C$, the least harmonic majorant of $s$ in $\Omega_{a, b}$ is $H_{a, b, s}$. If $(10)$ holds and $\psi\left(s^{+}\right)<\infty$, then $s$ has a harmonic majorant in $D_{a}$ and it is now clear that the least such majorant is $\lim _{b \rightarrow \infty} H_{a, b, s}$. By Theorem 2 (iii), (iv), this limit is given by (11).

\section{Proof of Theorem 4}

If the hypotheses of Theorem 4 are satisfied, then, by Theorem $2, s$ has a positive harmonic majorant in $D_{a}$. Hence, by Lemma E, $s$ has the representation (20) in $D_{a}$, and by Lemmas 3,4 and $5, \psi_{a}(s)=c_{n} k$. If we write $H=I_{a, \infty, \mu}$ and if $a^{\prime}>a$, then in $D_{a^{\prime}}$ we have $H=I_{a^{\prime}, \infty, H}$, as is well known. Hence, by Lemma 3, $\psi_{a^{\prime}}(H)=0$. Also $\psi_{a^{\prime}}(y-a)=$ $\psi_{a^{\prime}}\left(y-a^{\prime}\right)+\psi_{a^{\prime}}\left(a^{\prime}-a\right)=c_{n}$, by Lemma 5 and the special case of Lemma 3 in which the Poisson integral is a constant function. Hence to show that $\psi_{a^{\prime}}(s)=c_{n} k=\psi_{a}(s)$, it remains to prove that $\psi_{a^{\prime}}(u)=0$. Since $u$ is positive and superharmonic in $D_{a^{\prime}}$, we can apply Lemma $E$ to $-u$ to obtain the representation

$$
u(M)=I_{a^{\prime}, \infty, v}(M)+l\left(y-a^{\prime}\right)+w(M) \quad\left(M \in D_{a^{\prime}}\right)
$$

where $v$ is a non-negative measure on $\mathbf{R}^{n}, l$ is a non-negative constant and $w$ is a potential in $D_{a^{\prime}}$. From Lemmas 3,4 and 5, we have $\psi_{a^{\prime}}(u)=c_{n} l$. Since $u(M) \geqq l\left(y-a^{\prime}\right)$ in $D_{a^{\prime}}$, it follows that $\psi_{a}(u) \geqq l \psi_{a}(y-a)+l_{a}\left(a-a^{\prime}\right)=c_{n} l$, by Lemmas 5 and 3 (trivial case). By Lemma $4, \psi_{a}(u)=0$. Hence $l=0$, and therefore $\psi_{a}(u)=0$, as required.

\section{Examples}

We give two examples to show how our theorems break down if the condition on the growth of $s$ is relaxed. For simplicity, we work only with $n=1$ and $a=0$. A point of $\mathbf{R}^{2}$ is denoted by $(x, y)$. Let $\varepsilon$ be a positive number and define $h_{\varepsilon}$ in $\mathbf{R}^{2}$ by

$$
h_{e}(x, y)=e^{\varepsilon x} \sin (\varepsilon y)
$$

Then $h_{\varepsilon}$ is harmonic in $\mathbf{R}^{2}$. Define functions $s_{1}$ and $s_{2}$ in $\bar{D}_{0}$ by writing $s_{1}=\left|h_{\varepsilon}\right|$ and

$$
s_{2}(x, y)=h_{\varepsilon}(x, y) \quad(0 \leqq y<\pi / \varepsilon), s_{2}(x, y)=0 \quad(y \geqq \pi / \varepsilon) .
$$


Then $s_{1}$ and $s_{2}$ are subharmonic in $D_{0}$ and vanish on $\partial D_{0}$. Also,

$$
\lim _{M \rightarrow \infty} s_{j}(M) e^{-\lambda|M|}=0 \quad(j=1,2)
$$

for any $\lambda>\varepsilon$. (Recall that if $s \in \mathscr{S}_{0}$, then (3) holds for all positive $\lambda$.) Straightforward calculations give

$$
\begin{array}{cc}
\Psi_{0}\left(s_{1}, y\right)=\Psi_{0}\left(s_{2}, y\right)=\pi y^{-1} \sin (\varepsilon y)\left(\pi^{2}-\varepsilon^{2} y^{2}\right)^{-1} & (0<y<\pi / \varepsilon), \\
\Psi_{0}\left(s_{1}, y\right)=\infty(y>\pi / \varepsilon, y \neq \pi / \varepsilon, 2 \pi / \varepsilon, \ldots), \quad \Psi_{0}\left(s_{1}, y\right)=0 & (y=\pi / \varepsilon, 2 \pi / \varepsilon, \ldots), \\
\Psi_{0}\left(s_{2}, y\right)=0 \quad(y \geqq \pi / \varepsilon) . &
\end{array}
$$

Hence $\Psi_{0}\left(s_{1}, y\right)$ takes both finite and infinite values on $(0, \infty)$ and has no limit as $y \rightarrow \infty$. Thus the conclusions of Theorem 1 fail for $s_{1}$. On the other hand, $\Psi_{0}\left(s_{2}, y\right)$ is realvalued on $(0, \infty)$ and possesses a finite limit as $y \rightarrow \infty$, but $s_{2}$ does not possess a harmonic majorant in any half-space $D_{a}$ with $0 \leqq a<\pi / \varepsilon$. (If $s_{2}$ had a harmonic majorant in $D_{a}$ with $0<a<\pi / \varepsilon$, then we would have

$$
\int_{-\infty}^{\infty}\left(1+x^{2}\right)^{-1} s(x, a) d x<\infty
$$

([9], Theorem 3, which is false). Thus Theorem 2 fails with $s=s_{2}$.

\section{REFERENCES}

1. D. H. Armitage, On hyperplane mean values of subharmonic functions, J. London Math. Soc. (2) 22 (1980), 99-109.

2. F. T. Brawn, The Green and Poisson kernels for the strip $\left.\mathbf{R}^{n} \times\right] 0,1[, J$. London Math. Soc. (2) 2 (1970), 439-454.

3. F. T. BRAwn, The Poisson integral and harmonic majorization in $\left.\mathbf{R}^{n} \times\right] 0,1[, J$. London Math. Soc. (2) 3 (1971), 747-760.

4. F. T. Brawn, Positive harmonic majorization of subharmonic functions in strips, Proc. London Math. Soc. (3) 27 (1973), 261-289.

5. M. Brelor, Éléments de la théorie classique du potentiel (C.D.U., Paris, 1965).

6. A. DINGhas, Über positive harmonische Funktionen in einen Halbraum, Math. Z. 46 (1940), 559-570.

7. T. M. FLETT, On the rate of growth of mean values of holomorphic and harmonic functions, Proc. London Math. Soc. (3) 20 (1970), 749-768.

8. L. L. Helms, Introduction to Potential Theory (Wiley-Interscience, New York, 1969).

9. Ü. Kuran, Harmonic majorization in half-balls and half-spaces, Proc. London Math. Soc. (3) 21 (1970), 614-636. 
10. Ü. KURAN, On the half-spherical means of subharmonic functions in half-spaces, $J$. London Math. Soc. (2) 2 (1970), 305-317.

11. Ü. Kuran, A criterion of harmonic majorization in half-spaces, Bull. London Math. Soc. 3 (1971), 21-22.

12. S. Nualtaranee, On least harmonic majorants in half-spaces, Proc. London Math. Soc. (3) 22 (1973), 243-260.

13. M. Tsuj, Potential Theory in Modern Function Theory (Maruzen, Tokyo, 1959).

14. G. N. Watson, A Treatise on the Theory of Bessel Functions (C.U.P., Cambridge, 1922).

15. N. A. Watson, A limit function associated with harmonic majorization on half-spaces, $J$. London Math. Soc. (2) 9 (1974), 229-238.

The QUEen'S UNIVERSITY

BELFAST BT7 1NN 